\title{
The linear induction motor (LIM) \& single linear induction motor (SLIM)
}

\author{
Nahid Ahmadinia
}

M. A. Electrical Power Engineering, Science and Research Branch, Islamic Azad University, Broujerd, Iran

\section{Email address:}

Nahmadinia@yahoo.com

\section{To cite this article:}

Nahid Ahmadinia. The Linear Induction Motor (LIM) \& Single Linear Induction Motor (SLIM). American Journal of Electrical Power and Energy Systems. Vol. 3, No. 4, 2014, pp. 71-75. doi: 10.11648/j.epes.20140304.11

\begin{abstract}
First of all mentions linear induction machines in 1890, only two years after the discovery of the rotary induction principle. Basically the concept of the linear device consists in imagining a rotary machine to be cut along a radial plane and 'unrolled' so that the primary member then consists of a single row of coils in slots in a laminated steel core. The differences between rotary and linear motors are outlined and reasons for the slow application of linear motors are explained. Principal developments in linear machines since the 1950s are described. Induction motor which can be used to power capsules in an Xv capsules in a pneumatic capsule pipeline system. Several optimal design schemes of a single sided linear induction motor (SLIM) adopted in linear metro are presented in this paper Firstly the equivalent circuit of SLIM fully considering the end effects, half-filled slots, back iron saturation and skin effect is proposed ,based on one dimensional air gap magnetic equations In the circuit, several coefficients including longitudinal end effect coefficients $\operatorname{Kr}(\mathrm{s})$ and $\mathrm{Kx}(\mathrm{s})$, transversal end edge effect coefficients $\mathrm{Cr}(\mathrm{s})$ and $\mathrm{Cx}(\mathrm{s})$, and skin effect coefficient $\mathrm{K}$ fare achieved by using the dummy electric potential method and complex power equivalence between primary and secondary sides Furthermore, several optimal design restraint equations of SLIM are provided in order to improve the operational efficiency and reduce the primary weight. The result tries to establish a new concept for elevators through a new construction technique and assembly of the system with counterweight, which increases the reliability and comfort with cost reduction.
\end{abstract}

Keywords: Electric Motors, linear Induction Motor (LIM), Single-Sided linear (SLIM)

\section{Introduction}

The SLIM has the following merits comparing with the rotary induction motor (RIM) : greater ability to exert thrust on the secondary without mechanical contacts, higher acceleration or deceleration, less wear of the wheels, smaller turn circle radius, and more flexible road line. Because of its cut-open magnetic circuit, the linear induction motor (LIM) possesses the inherent characteristics such as longitudinal end-effect, transversal edge-effect and normal force. In addition, it also has half-filled slots in the primary ends. Therefore, an accurate equivalent circuit model of LIM is difficult to be obtained compared to that of RIM. Many analysis techniques of SLIM have been studied and developed in the past years. However, effective methods on the design scheme of SLIM have not been obtained due to the following reasons. The selection of electric loading and magnetic loading by loading distribution is so difficult that one cannot calculate the apparent power (kVA) easily. The
Power factor and efficiency are affected by the end effect which is again affected by the design technique of SLIM. It uses a rotary electrical motor as source of motion in order to convert the rotary mot ion into a linear motion. Often, it is necessary to use a complex mechanical system of gears, axles and screws jacks. When used directly, these transmission systems for movement have great losses. Among the reasons is the increased an abrasive wear due to the friction of the mechanical parts, even when using low viscosity fluids for the lubrication. This results into higher operational and maintenance costs Therefore, for transport applications, the use of an electrical machine that produces directly the linear motion would result in lowers operational and maintenance costs as well as higher reliability and efficiency. The purpose of this paper is to propose mover position control of linear induction motor (LIM) using an adaptive backstopping approach based on field orientation. 


\section{History}

The history of linear electric motors can be traced back at least as far as the 1840 s, to the work of Charles Wheatstone at King's in London, but Wheatstone's model was too inefficient to be practical. In 1824, the French physicist François Arago formulated the existence of rotating magnetic fields, termed Arago's rotations, which, by manually turning switches on and off, Walter Baily demonstrated in 1879 as in effect the first primitive induction motor. Practical alternating current induction motors seem to have been independently invented by Galileo Ferraris and Nikola Tesla, a working motor model having been demonstrated by the former in 1885 and by the latter in 1887. In April 1888, the Royal Academy of Science of Turin published Ferraris's research on his AC polyphone motor detailing the foundations of motor operation. George Westinghouse, who was developing an alternating current power system at that time, licensed Tesla's patents in 1888 and purchased a US patent option on Ferraris' induction motor concept. Tesla was also employed for one year as a consultant. Westinghouse employee C. F. Scott was assigned to assist Tesla and later took over development of the induction motor at Westinghouse. Steadfast in his promotion of three-phase development, Mikhail Dolivo-Dobrovolsky's invented the cage-rotor induction motor in 1889 and the three-limb transformer in 1890. The General Electric Company (GE) began developing three-phase induction motors in 1891. By 1896, General Electric and Westinghouse signed a cross-licensing agreement for the bar-winding-rotor design, later called the squirrel-cage rotor.

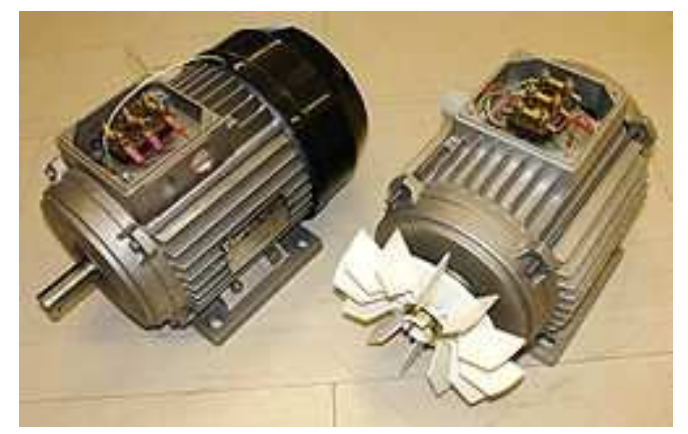

Figure 1. Three-phase totally enclosed fan-cooled (TEFC) induction motor with and, at right, without end cover to show cooling fan. In TEFC motors, interior losses are dissipated indirectly through enclosure fins mostly by forced air convection

\section{Linear Electric Motor \& Linear Induction Motor}

A linear electric motor's primary typically consists of a flat magnetic core (generally laminated) with transverse slots which are often straight cut with coils laid into the slots, with each phase giving an alternating polarity and so that the different phases physically overlap. The secondary is frequently a sheet of aluminum, often with an iron backing plate. Some LIMs are double sided, with one primary either side of the secondary, and in this case no iron backing is needed. Two sorts of linear motor exist, short primary, where the coils are truncated shorter than the secondary, and a short secondary where the conductive plate is smaller. Short secondary LIMs are often wound as parallel connections between coils of the same phase, whereas short primaries are usually wound in series

The primaries of transverse flux LIMs have a series of twin poles lying transversely side-by-side, with opposite winding directions. These are typically made either with a suitably cut laminated backing plate or a series of transverse U-cores .Induction motors use electromagnetic induction to rotate a shaft or rotor and create mechanical energy from electric energy. With minimal design alterations an electric motor like this also has the ability to create electricity from mechanical energy, although these motors are referred to as generators. Electromagnetic induction is the production of an electrical potential difference (or Voltage) across a conductor situated in a changing magnetic field. A Faradays Law state that electromotive force (EMF) produced along a closed path is proportional to the rate of change of the magnetic flux through any surface bounded by that path. In practice, this means that an electrical current will flow in any closed conductor, when the magnetic flux through a surface bounded by the conductor changes. This applies whether the field itself changes in strength or the conductor is moved through it.

The most basic Induction motor is the Squirrel cage motor. Squirrel cage motors are the most common industrial $\mathrm{AC}$ motor, when running off a constant AC supply they are simply constant speed devices. They acquired there name from the rotors used which resemble a squirrel running cage. This type of motor has revolutionized the way factories operate, improving efficiency and safety compared to earlier mechanical methods.

When a squirrel cage motor is used with a three-phase power source, it forms the simplest electric motor. This motor is made up of three fixed coils around a rotating shaft which consists of a core filled with a series of conductors set up in a circle pattern around the shaft. With the three-phase current flowing through the coils a rotating magnetic field is produced, the field then induces a current in the conductors on the shaft. This is where three-phase power outperforms single phase. For the shaft to rotate smoothly without stopping the three coils situated at $120^{\circ}$ from each other are supplied with power at precise intervals. This can be better described by the graphics below.

Usually, transportation of laminated products is done through rolls, put in rotation by electric motors. The acting moving force depends solely on the adherence of the laminated product to the moving rolls, which reduces the speed of the latter. Since a large acceleration is not possible due to the resulting high inertial forces, a sliding of the bars on the rolls will occur. A solution to this problem may be obtained by using a linear asynchronous motor whose moving body would be the laminated product.

The experiments were performed under various practical 
conditions and changes were made in the system in order to practically obtain an optimal design of the model over which the experiments were carried on. The experimental data were obtained by changing the various parameters of the LIM such as air gap, magnetic gap, changing the materials of the secondary of LIM, by creating breaks in the electrical and magnetic circuits of the LIM, by de-aligning the primary with respect to secondary etc.by using different supply and controls. The tests were successfully conducted for direct voltage control, frequency control and variable voltage variable frequency control i.e. V/f control by using VVVF Drive and three phase auto Transformer. The data taken after experiments are plotted individually and relatively in order to compare the results under different situations finally, the observations made and the graphs plotted have been explained and results successfully approved with the practical requirements for Traction Applications of Linear Induction Motor.

A linear electric motor's primary typically consists of a flat magnetic core (generally laminated) with transverse slots which are often straight cut with coils laid into the slots, with each phase giving an alternating polarity and so that the different phases physically overlap. The secondary is frequently a sheet of aluminum, often with an iron backing plate. Some LIMs are double sided, with one primary either side of the secondary, and in this case no iron backing is needed.

Two sorts of linear motor exist, short primary, where the coils are truncated shorter than the secondary, and a short secondary where the conductive plate is smaller. Short secondary LIMs are often wound as parallel connections between coils of the same phase, whereas short primaries are usually wound in series. The primaries of transverse flux LIMs have a series of twin poles lying transversely side-by-side, with opposite winding directions. These are typically made either with a suitably cut laminated backing plate or a series of transverse U-cores. For this reason, induction motors are sometimes referred to as asynchronous motors. An induction motor can be used as an induction generator, or it can be unrolled to form a linear induction motor which can directly generate linear motion.

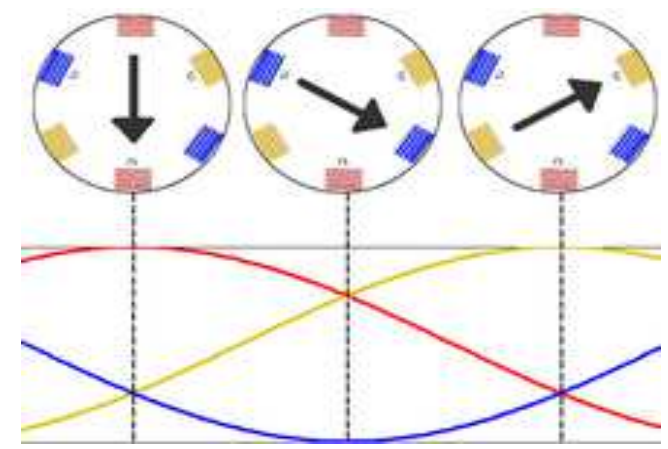

Figure 2. A three-phase power supply provides a rotating magnetic field in an induction motor.

In both induction and synchronous motors, the $\mathrm{AC}$ power supplied to the motor's stator creates a magnetic field that rotates in time with the $\mathrm{AC}$ oscillations. Whereas a synchronous motor's rotor turns at the same rate as the stator field, an induction motor's rotor rotates at a slower speed than the stator field. The induction motor stator's magnetic field is therefore changing or rotating relative to the rotor. This induces an opposing current in the induction motor's rotor, in effect the motor's secondary winding, when the latter is short-circuited or closed through external impedance. The rotating magnetic flux induces currents in the windings of the rotor in a manner similar to currents induced in a transformer's secondary winding(s). The currents in the rotor windings in turn create magnetic fields in the rotor that react against the stator field.

\section{Synchronous Speed}

An AC motor's synchronous speed, $n_{s}$, is the rotation rate of the stator's magnetic field, which is expressed in revolutions per minute as

$$
n_{s}=\frac{120 \times f}{p}
$$

where $f$ is the motor supply's frequency in Hertz and $p$ is the number of magnetic poles. That is, for a six-pole three-phase motor with three pole-pairs set $120^{\circ}$ apart, $p$ equals 6 and $n_{s}$ equals 1,000 RPM and 1,200 RPM respectively for $50 \mathrm{~Hz}$ and $60 \mathrm{~Hz}$ supply systems.

\subsection{Slip}

Slip, $s$, is defined as the difference between synchronous speed and operating speed, at the same frequency, expressed in rpm or in percent or ratio of synchronous speed. Thus

$$
s=\frac{n_{s}-n_{r}}{n_{s}}
$$

where $n_{s}$ is stator electrical speed, $n_{r}$ is rotor mechanical speed. Slip, which varies from zero at synchronous speed and 1 when the rotor is at rest, determines the motor's torque. Since the short-circuited rotor windings have small resistance, a small slip induces a large current in the rotor and produces large torque. At full rated load, slip varies from more than $5 \%$ for small or special purpose motors to less than $1 \%$ for large motors. These speed variations can cause load-sharing problems when differently sized motors are mechanically connected. Various methods are available to reduce slip, VFDs often offering the best solution.

\section{Principles}

In this model of electric motor, the force is produced by linearly moving magnetic field acting on conductors in the field. Any conductor is it a loop, a coil or simply a piece of plate metal that is placed in this field will have eddy currents induced in it thus creating an opposing magnetic field, in accordance with Lenz's law. The two opposing fields will repel each other, thus creating motion as the magnetic field sweeps through the metal: 


$$
n_{s}=2 f_{s} / p
$$

Where, $f_{s}$ is supply frequency in $\mathrm{Hz}, \mathrm{p}$ is the number of poles, and $n_{s}$ is the synchronous speed of the magnetic field in revolutions per second.

The travelling field pattern has a velocity of:

$$
v_{s}=2 t f_{s}
$$

$v_{s}$ Is velocity of the linear travelling field in $\mathrm{m} / \mathrm{s}, \mathrm{t}$ is the pole pitch.

For a slip of s, the speed of the secondary in a linear motor is given by

$$
v_{r}=(1-s) v_{s}
$$

\section{Thrust}

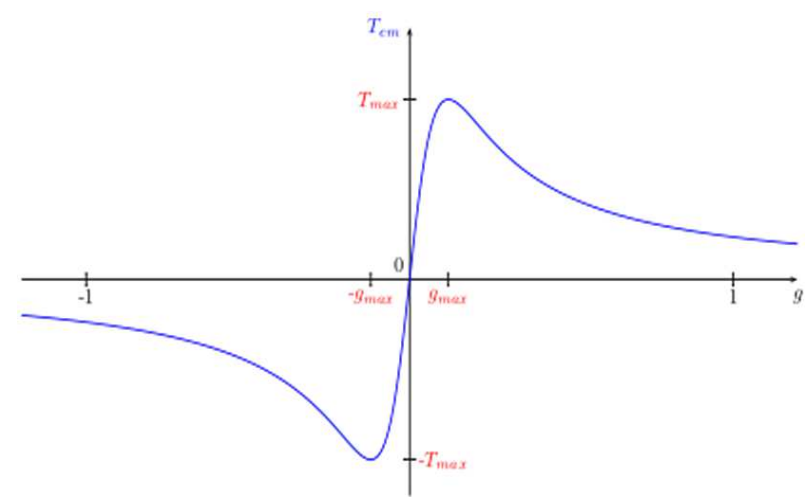

Figure 3. Thrust generated as a function of slip

As explained earlier, the input power to the stator windings is utilized in producing useful mechanical power which is exerted on the rotor and to account for the rotor copper losses. In terms of the equivalent circuit com ponents, the mechanical power developed by the rotor is the power transferred across the air-gap from the stator to rotor minus the rotor copper loss. Under normal operations, the LIM develops a thrust proportional to the square of the applied voltage, and this reduces as slip is reduced similarly to that of an induction motor with a high rotor resistance.

\subsection{Levitation}

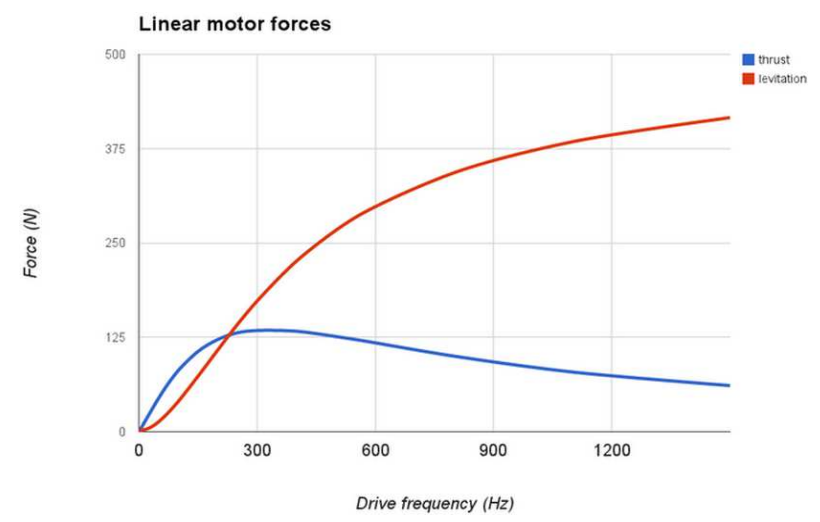

Figure 4. Levitation and thrust force curves of a linear motor
In addition, unlike a rotary motor, an electro dynamic levitation force is shown, this is zero at zero slip, and gives a roughly constant amount of force/gap as slip increases in either direction. This occurs in single sided motors, and levitation will not usually occur when an iron backing plate is used on the secondary, since this causes an attraction that overwhelms the lifting force. A linear induction motor (LIM) is an AC asynchronous linear motor that works by the same general principles as other induction motors but is very typically designed to directly produce motion in a straight line.

linear induction motors have a finite length primary, which generates end-effects, whereas with a conventional induction motor the primary is arranged in an endless loop. Despite their name, not all linear induction motors produce linear motion; some linear induction motors are employed for generating rotations of large diameters where the use of a continuous primary would be very expensive. As with rotary motors, linear motors frequently run on a 3 phase power supply and can support very high speeds. However, there are end-effects which reduce the force, and it's often not possible to fit a gearbox to trade off force and speed. Linear induction motors are thus frequently less energy efficient than normal rotary motors for any given required force output. LIMs are often used where contactless force is required, where low maintenance is desirable, or where the duty cycle is low. Their practical uses include magnetic levitation, linear propulsion, and linear actuators. They have also been used for pumping liquid metals.

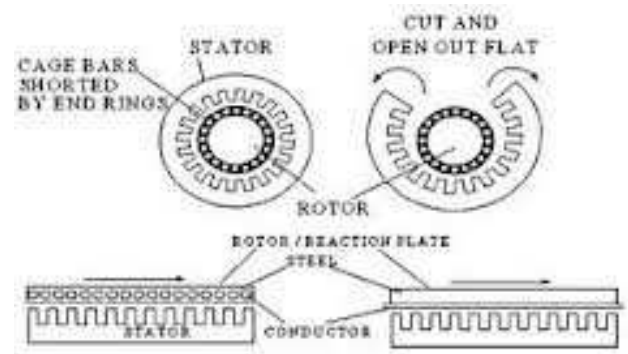

Figure 5. Cutting and Unrolling a RIM to a LIM

This paper is interested in thrust and its relation to other variable parameters. The normal force is perpendicular to the stator in the z-direction. Lateral forces are undesirable forces which are developed in a SLIM because of the orientation of the stator. These forces are a matter of concern in high frequency operation $(60 \mathrm{~Hz})$ where they increase in magnitude. A set of guided mechanical wheel tracks is sufficient to eliminate a small lateral force. For the analysis and design of a SLIM having negligible end-effects perphase conventional equivalent circuit shown in Fig 1 may be used. The circuit components are determined from the SLIM parameters. The SLIM performances to be determined are thrust and efficiency. The approximate equivalent circuit of a LIM is presented as shown in Fig 1. This circuit is on a per phase basis. The core losses are neglected because a realistic air gap flux density leads to mode rate flux densities in the core and hence, rather low core losses. Skin effect is small at 
rated frequency for a flat linear induction motor with a thin conductive sheet on the secondary. Therefore, equivalent rotor inductance is negligible. The remaining non-negligible parameters are shown in Fig 1 and are discussed blew. The drive generated by linear induction motors is somewhat similar to conventional induction motors; the drive forces show a roughly similar characteristic shape relative to slip, albeit modulated by end effects. Unlike a circular induction motor, a linear induction motor shows 'end effects'. These end effects include losses in performance and efficiency that are believed to be caused by magnetic energy being carried away and lost at the end of the primary by the relative movement of the primary and secondary. With a short secondary, the behavior is almost identical to a rotary machine, provided it is at least two poles long, but with a short primary reduction in thrust occurs at low slip. However, because of end effect, linear motors cannot 'run light'normal induction motors are able to run the motor with a near synchronous field under low load conditions. Due to end effect this creates much more significant losses with linear motors.

\section{The Basic Principle of LIM Operation}

The principle of operation of a LIM is the same as that of a rotary induction motor. A linear Induction motor is basically obtained by opening the rotating squirrel cage induction motor and laying it flat. This flat structure produces a linear force instead of producing rotary torque from a cylindrical machine. LIMs can be designed to produce thrust up to several thousands of Newton's. The winding design and supply frequency determine the speed of a LIM. The basic principle of LIM operation is similar to that of a conventional rotating squirrel-cage induction motor. Stator and rotor are the two main parts of the conventional three phase rotary induction motor. The stator consists of a balanced poly phase winding which is uniformly placed in the stator slots along its periphery. The stator produces a sinusoid ally distributed magnetic field in the air-gap rotating at the uniform speed $2 \omega / \mathrm{p}$, with $\omega$ representing the network pulsation (related to the frequency f by $\omega=2 \pi \mathrm{f}$ ) and (p) the number of poles. The relative motion between the rotor conductors and the magnetic field induces a voltage in the rotor. This induced voltage will cause a current to flow in the rotor and will generate a magnetic field. The interaction of these two magnetic fields will produce a torque that drags the rotor in the direction of the field. This principle would not be modified if the squirrel cage were re placed by a continuous sheet of conducting.

\section{Conclusion}

In this Paper, the best way to cover a vast range of the rotor speed was found by defining an efficiency criterion at which the system would reach the steady state before certain time, which in this case was $2 \mathrm{~s}$. This was developed as a first stage in the control of the LIM, where setting a desired speed of the rotor and reaching it rapidly were the main concerns. In order to achieve this compromise of reaching the reference speed in a small time was found that the best way was not only by changing the frequency of the source but also the voltage amplitude. For that purpose two multiplexer blocks were implemented in the simulation model. The first one addressed the look up table corresponding to the range under which the speed reference of the rotor was covered outputting the optimum value of frequency. The second multiplexer addressed the value of voltage amplitude corresponding to the speed reference of the rotor (upon defined ranges) necessary for the system to reach the desired conditions.

\section{References}

[1] Chapman, S. (1999). Electric Machinery Fundamentals, New York: McGraw-Hill.

[2] Control Techniques. (2007). User Guide Unidrive Models size 0 to 6 . Emerson Industrial Automation

[3] Doyle, M. Electromagnetic Aircraft Launch System EMALS. Naval Air Warfare Center, Aircraft Division Lakehurst, NJ 08733

[4] Meeker D. Indirect Vector Control of a Redundant Linear Induction Motor for Aircraft Launch. NAVAIR Public Release 08-642.

[5] Nowak, L. (2001). Movement simulation in 3D eddy current transient problem COMPEL: The International Journal for Computation and Mathematics in Electrical and Electronic Engineering, Vol. 20, No. 1, 2001, pp. 293 - 302, MCB University Press.

[6] Ogata, K. (2002). Modern Control Engineering, New Jersey: Prentice Hall.

[7] Roa, C. (2010). Electric Motor Control System with Application to Marine Propulsion, MSc Thesis Dissertation, Department of Ocean and Mechanical Engineering, Florida Atlantic University, Florida.

[8] Upadhyay, J. Mahendra S.N, Electric Traction, Allied publishers Ltd, New Delhi, 2000.

[9] Xiros, N. (2010). Nonlinear Modeling of Linear Induction Machines for Analysis and Control of Novel Electric Warship Subsystems 